\title{
Oesophageal Metastasis from Colorectal Cancer
}

\author{
Sarah C. Thomasset Giuseppe Garcea David P. Berry \\ Department of Hepatobiliary and Pancreatic Surgery, Leicester General Hospital, \\ Leicester, UK
}

\section{Key Words}

Colorectal cancer · Metastases · Oesophagus

\begin{abstract}
Metastasis to the oesophagus is most frequently described in association with lung or breast cancer. Diagnosis is frequently complicated as often only normal tissue is present in endoscopic biopsy specimens. Although oesophagectomy for metastasis has been described, few patients are suitable for curative resection. We report the case of a 62year-old man who developed an oesophageal metastasis from colorectal cancer and review the available literature.
\end{abstract}

\section{Introduction}

Metastasis to the oesophagus is rare. The predominantly submucosal focus of the disease means tumour is frequently absent from endoscopic biopsy specimens [1-3] and a high degree of suspicion is required to establish the correct diagnosis. Oesophageal metastases may be mistaken for a number of conditions with markedly different treatments, including benign oesophageal strictures and primary oesophageal carcinoma [1]. Increased awareness of the condition may prevent misdiagnosis.

\section{Case Report}

A 62-year-old man underwent a sigmoid colectomy for Dukes C carcinoma. Histological examination revealed a moderately differentiated tumour with extramural vascular invasion and involvement of two out of the nineteen lymph nodes resected. Adjuvant chemotherapy was not offered in view of multiple co-morbidities including chronic renal failure, diabetes, ischaemic heart disease and hypertension. A routine computer tomography (CT) scan performed a year after the sigmoid colectomy revealed metastatic disease in the right liver, caudate lobe and segment IV. A subsequent thoracic CT scan failed to show lung metastases, however, a polypoidal lesion was identified in the lower oesophagus (fig. 1). A positron emission tomography scan showed areas of abnormal uptake of radioisotope in the liver, lower oesophagus and left gastric nodes (fig. 2). The patient denied dysphagia but underwent oesophagogastroscopy which revealed a soft tissue mass in the lower oesophagus, the appearance being 
consistent with oesophageal carcinoma. Biopsies demonstrated adenocarcinoma which was histologically identical to the primary sigmoid lesion. The patient commenced treatment with 5fluorouracil and folinic acid but died of progressive disease six months after metastases were diagnosed.

\section{Discussion}

Lymphatic and blood-borne metastases to the oesophagus are uncommon. Since the first case of oesophageal metastasis from prostate cancer was reported in 1942 [4], metastases from a number of other primary sites, most frequently breast and lung, have been described $[2,3]$. To date there are two case reports describing oesophageal metastases from colorectal cancer [5, 6]. Despite clinical rarity, autopsy studies have revealed oesophageal metastases in up to $6.1 \%$ of individuals with malignant disease and are particularly common in those with lung, breast, tongue, uterine and stomach malignancies. The majority of oesophageal metastases discovered at autopsy are only detected by microscopic examination (68\%) [1].

Oesophageal metastases may present with dysphagia or upper gastrointestinal bleeding [2]. Occasionally dysphagia due to oesophageal metastases can occur as a presenting symptom in patients with previously undiagnosed malignant disease, usually lung cancer [3]. More commonly patients present with symptoms of oesophageal metastases months to years after a primary tumour has been treated. Periods in excess of 20 years between initial treatment and presentation with oesophageal metastases have been described [7].

Diagnosis of oesophageal metastases at endoscopy can be difficult. In some patients an ulcer or protruding tumour may be evident and indistinguishable from primary oesophageal carcinoma $[1,6]$. However, endoscopy characteristically reveals an oesophageal stricture associated with macroscopically normal mucosa, resembling a benign stricture. As disease is focused in the submucosa it is not uncommon for tumour to be absent from endoscopic biopsies [1-3]. Endoscopic ultrasound (EUS) is a useful adjunct possibly revealing the presence of tumour or lymphadenopathy not apparent on standard endoscopy and acting as a guide for obtaining biopsies [2, 8]. Oesophageal metastases typically cause a smooth concentric stricture on barium swallow [1]. Less frequently appearances may mimic oesophageal carcinoma or benign pathology such as achalasia [5]. Concentric thickening of the oesophageal wall overlying a stricture but not associated with any mass extrinsic to the oesophagus is the usual finding on CT [2].

Oesophagectomy has been reported for the treatment of oesophageal metastases originating from the breast, lung and ovary [1]. A maximum survival of 14 years has been described following oesophagectomy for metastases originating from ovarian cancer previously treated by bilateral salpingo-oophorectomy [1]. However, most patients with metastasis to the oesophagus already have metastases elsewhere and the treatment of choice is therefore chemotherapy, radiotherapy and/or hormone therapy. Endoscopic dilatation and stenting may be used to palliate dysphagia but should be approached with caution as perforation in the presence of metastases has been described [9].

In this case oesophageal metastases were recognised at an early stage following oesophagogastroscopy and biopsy. However, disease is predominantly submucosal and biopsies are often normal, which complicates diagnosis.

Oesophageal metastasis, although rare, should be considered in patients presenting with oesophageal pathology where there is a previous history of malignant disease. 
Fig. 1. Thoracic CT scan showing a polypoidal lesion in the lower oesophagus (black arrow).

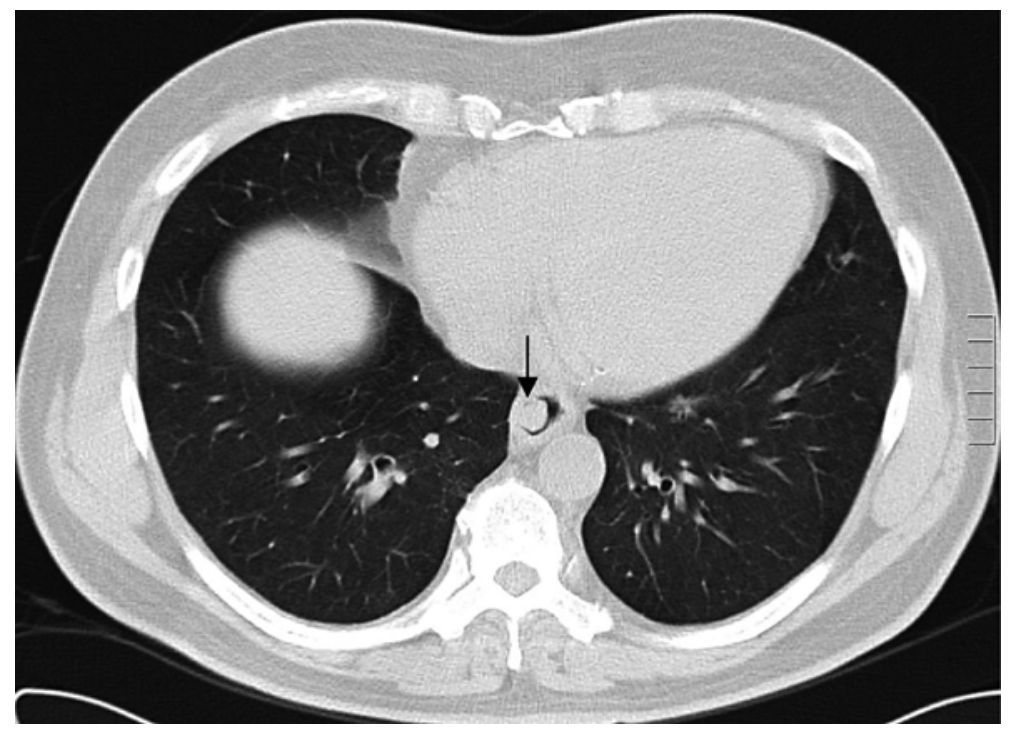

Fig. 2. Positron emission tomography scan showing increased uptake of radioisotope in the oesophagus and liver.

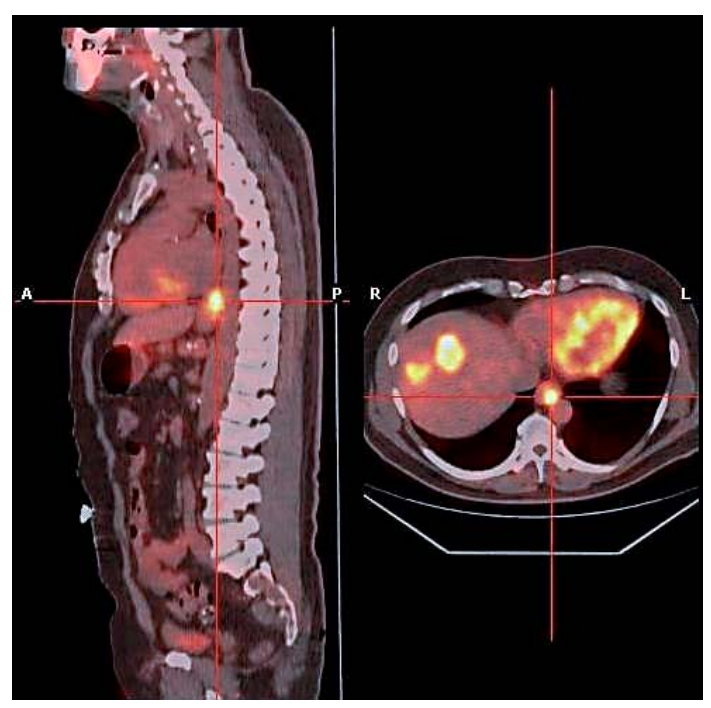




\section{References}

1 Mizobuchi S, Tachimori Y, Kato H, Watanabe H, Nakanishi Y, Ochiai A: Metastatic esophageal tumors from distant primary lesions: Report of three esophagectomies and study of 1835 autopsy cases. Jpn J Clin Oncol 1997;27:410414.

-2 Simchuk EJ, Low DE: Direct esophageal metastasis from a distant primary tumor is a submucosal process: A review of six cases. Dis Esophagus 2001;14:247-250.

-3 Anderson MF, Harell GS: Secondary esophageal tumors. Am J Roentgenol 1980;135:1243-1246.

4 Gross P, Freedman LJ: Obstructing secondary carcinoma of the esophagus. Arch Pathol 1942;33:361-364.

5 Fischer MS: Metastasis to the esophagus. Gastrointest Radiol 1976;1:249-251.

6 Lohsiriwat V, Boonnuch W, Suttinont P: Esophageal metastasis from rectal carcinoma. J Clin Gastroenterol 2005;39:744.

-7 Sunada F, Yamamoto H, Kita H, Hanatsuka K, Ajibe H, Masuda M, Hirasawa T, Osawa H, Sato K, Hozumi Y, Sugano K: A case of esophageal stricture due to metastatic breast cancer diagnosed by endoscopic mucosal resection. Jpn J Clin Oncol 2005;35:483-486.

-8 Faigel DO, Deveney C, Phillips D, Fennerty MB: Biospy-negative malignant esophageal stricture: Diagnosis by endoscopic ultrasound. Am J Gastroenterol 1998;93:2257-2260.

-9 Anaya DA, Yu M, Karmy-Jones R: Esophageal perforation in a patient with metastatic breast cancer to the esophagus. Ann Thorac Surg 2006;81:1136-1138. 